\title{
Fully embedded myoelectric control for a wearable robotic hand orthosis
}

\section{Conference Paper}

Author(s):

Ryser, Franziska; Butzer, Tobias; Held, Jeremia P.; Lambercy, Olivier; Gassert, Roger (D)

Publication date:

2017-07

Permanent link:

https://doi.org/10.3929/ethz-b-000228392

Rights / license:

In Copyright - Non-Commercial Use Permitted

Originally published in:

https://doi.org/10.1109/icorr.2017.8009316 


\title{
Fully Embedded Myoelectric Control for a Wearable Robotic Hand Orthosis
}

\author{
Franziska Ryser*1, Tobias Bützer*1 ${ }^{*}$, Jeremia P. Held ${ }^{2}$, Olivier Lambercy ${ }^{1}$ and Roger Gassert ${ }^{1}$
}

\begin{abstract}
To prevent learned non-use of the affected hand in chronic stroke survivors, rehabilitative training should be continued after discharge from the hospital. Robotic hand orthoses are a promising approach for home rehabilitation. When combined with intuitive control based on electromyography, the therapy outcome can be improved. However, such systems often require extensive cabling, experience in electrode placement and connection to external computers. This paper presents the framework for a stand-alone, fully wearable and real-time myoelectric intention detection system based on the Myo armband. The hard and software for real-time gesture classification were developed and combined with a routine to train and customize the classifier, leading to a unique ease of use. The system including training of the classifier can be set up within less than one minute. Results demonstrated that: (1) the proposed algorithm can classify five gestures with an accuracy of $98 \%$, (2) the final system can online classify three gestures with an accuracy of $94.3 \%$ and, in a preliminary test, (3) classify three gestures from data acquired from mildly to severely impaired stroke survivors with an accuracy of over $78.8 \%$. These results highlight the potential of the presented system for electromyography-based intention detection for stroke survivors and, with the integration of the system into a robotic hand orthosis, the potential for a wearable platform for all day robotassisted home rehabilitation.
\end{abstract}

\section{INTRODUCTION}

With over 14'000 cases every day worldwide, stroke is the leading cause for chronic hand impairment [1], [2]. In most cases, prescribed physical and occupational therapy lead to improvement of the motor and sensory function of the affected limb [3]. However, after completing inpatient therapy, many subjects remain with limited residual hand function, impeding the use of their hand in activities of daily living at home. The lower the residual hand function, the more a patient is prone to learned non-use [4], i.e. decreased use of the affected limb because of its low functionality. To prevent learned non-use and enhance residual hand function, the rehabilitative training needs to be continued after discharge from the hospital [5].

Robotic systems can provide a platform for home rehabilitation and for continued therapy, independent from scheduled sessions at the hospital. Aiming at home rehabilitation [6], [7], state of the art robotic hand orthoses offer continuous assistance-as-needed or are combined with video games and performance feedback to increase the

\footnotetext{
* These authors contributed equally to this work.

1 The authors are with the Rehabilitation Engineering Laboratory, Department of Health Sciences and Technology, ETH Zurich, Switzerland tobias.buetzerahest.ethz.ch

2 The author is with the Zurich University Hospital, Department of Neurology, University of Zurich, Switzerland
}

motivation of the patient [8], as the Gloreha (Idrogenet srl., Lumezzane, Italy) or Hand of Hope (Rehab-Robotics Ltd., Hong Kong). Using intention detection is hypothesized to stimulate Hebbian plasticity [9] and thus, to be beneficial for neurorehabilitation [10]. Various intention detection methods can be integrated into robotic hand orthoses, triggering the desired robot motion based on users' biosignal. Methods such as electroencephalography (EEG) [11], electrooculography (EOG) [12] and near-infrared spectroscopy (NIRS) [13] have been used in 2-class problems such as the differentiation between two grasp patterns, or for on/off control of robotic devices like hand orthoses. However, all approaches are highly limited in the number of classes that can be differentiated. In order to cover the large variety in human grasp types, several gestures have to be recognized [14]. Surface electromyography (sEMG) based algorithms have been used to classify up to 53 grasp types at $61.5 \%$ overall accuracy [15]. However, EMG systems typically use multiple electrodes [15]-[17] or electrode arrays [18], which require cumbersome cabling, conductive gels and experience in electrode placement, making them hardly applicable in a home rehabilitation scenario. The commercially available Myo armband (Thalmic Labs, Kitchener, Ontario, Canada) is a lightweight and easy to use alternative to state of the art EMG data acquisition systems. Its eight dry EMG electrodes do not require exact placement and its built-in classifier can detect six different hand gestures. The Myo armband has therefore been used as alternative EMG data acquisition system for the control of robotic hand prostheses [19]. However, the built-in classifier is only suitable for very specific, hard-coded gestures. Moreover, the Myo armband requires connection with an external computer at all times.

In this paper we present the framework for a stand-alone, real-time and wearable EMG intention detection system based on the Myo armband. In contrast to previous work, the system does not require any connection to an external computer and can be trained to identify custom gestures in a short training routine after the system is donned. The classifier training routine can be executed before each use of the system and therefore enables much higher intersession classification accuracies compared to systems with pre-trained classifiers. Furthermore, the system can fully integrate robotic systems, such as the hand orthosis presented in [20] creating a platform for all day home rehabilitation.

\section{Methods}

For intention detection based control, EMG signal patterns are recorded with the Myo armband and mapped to selected 


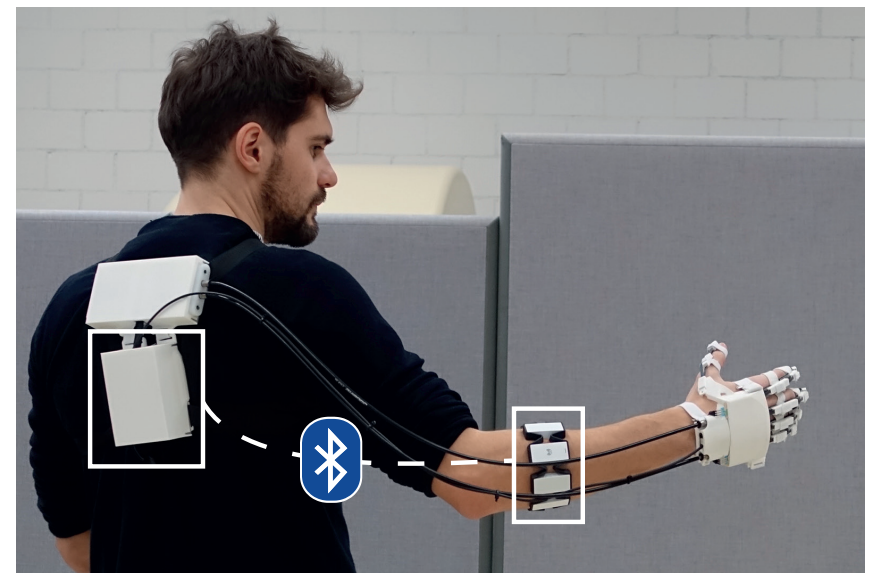

Fig. 1. System overview showing the Myo armband and the hand orthosis with a backpack containing the control unit (microcontroller and printed circuit board), and the electronics box above (motors and battery). The motors and the hand orthosis are connected with Bowden cables. The Myo armband communicates wirelessly via Bluetooth Low Energy with the control unit.

gestures. This information can subsequently be used to trigger the position control of an assistive device, such as the two degrees of freedom (DOF) hand orthosis earlier presented in [20]. To decouple the system from stationary devices the EMG signal is transmitted via Bluetooth to an integrated control unit, where the signal classification is executed (Fig. 1).

\section{A. Requirements}

As desired grasp types may change for different therapy sessions and as EMG changes for every user and every recording session [21], a custom classifier is required. It has to detect up to 5 different hand gestures with an accuracy of about $95 \%$ to account for the variety in human grasp types [22]. To be used in activities of daily living, it has to detect the gestures reliably even when the arm is held in another configuration [23]. The different gestures should ideally be detected within 250-300ms (minimal perceivable delay) after the EMG signal is evoked, in order to maximize the user acceptance of the system [24]. To be compatible with a wearable robotic device, the EMG signal acquisition and classification has to be computed on a stand-alone and wearable device that provides the computational power and memory resources to process the EMG data with minimal delay. The intention detection system should be wearable for several hours a day. Therefore, the classification system and the EMG sensors have to be comfortable, suitable for patients with impaired hand function and easy to use for stroke survivors with cognitive deficits.

\section{B. Hardware choices}

We chose the Myo armband (Fig 2) [25] as data acquisition system due to its ease of use and wearing comfort. It combines eight dry surface EMG sensors and communicates via Bluetooth Low Energy (BLE). The EMG signal is filtered

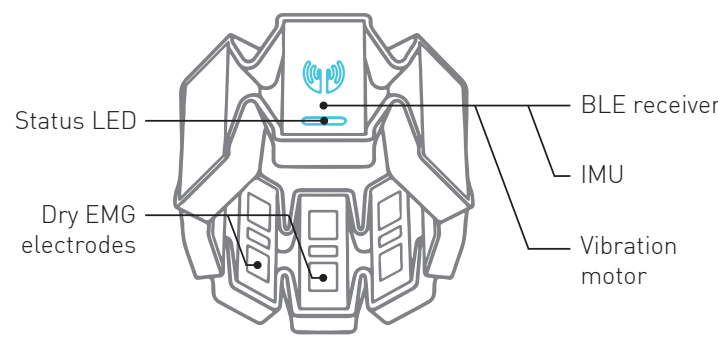

Fig. 2. The Myo armband from Thalmic Labs, containing a nineaxis inertial measurement unit (IMU), eight dry surface EMG sensors and vibration actuator for haptic feedback. Figure adapted from https://developer.thalmic.com.

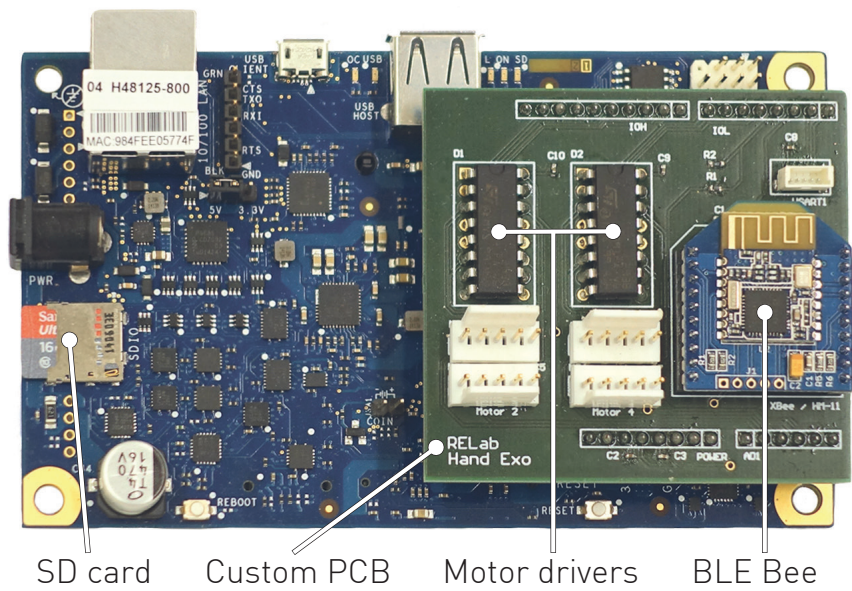

Fig. 3. The Galileo Gen2 development board by Intel with a custom printed circuit board (PCB). The microcontroller processes and classifies the EMG signal and controls the motors of the hand exoskeleton. The program developed in the Arduino IDE is stored on a micro-SD card. The PCB includes four motor drivers, pins for four motor cables, a Bluetooth module and a connector for an additional serial line to external sensors. Overall size of the electronics board: $123.8 \mathrm{~mm} \times 72 \mathrm{~mm} \times 32 \mathrm{~mm}$

against powerline noise interference and streamed with up to $200 \mathrm{~Hz}$ to a computer.

For computing the EMG signal, we chose the Galileo Gen2 development board from Intel. The Galileo Gen2 meets the requirements in terms of computational resources, with a 32-bit Intel Pentium processor, 512 kbytes SRAM and 256 Mbytes DRAM. Besides a Linux operating system, it supports the Arduino Software (IDE). As the Galileo Gen2 does not store the sketch internally, we booted the board from an external microSD card with an embedded Yocto linux image. Best results were achieved with the uClibc based Yocto image provided by Intel. The Bluetooth receiver was included in a custom-made printed circuit board and accessed by the digital input/output pins of the Galileo Gen2 (Fig. 3).

\section{Data acquisition}

The EMG signal is acquired and preprocessed by the Myo armband. To receive the data, a BLE Bee (HM-11 Bluetooth module on an XBee compatible form factor [26]) from Seeedstudio was connected via UART to the Galileo Gen2 board. This allows to exchange the BLE Bee with 


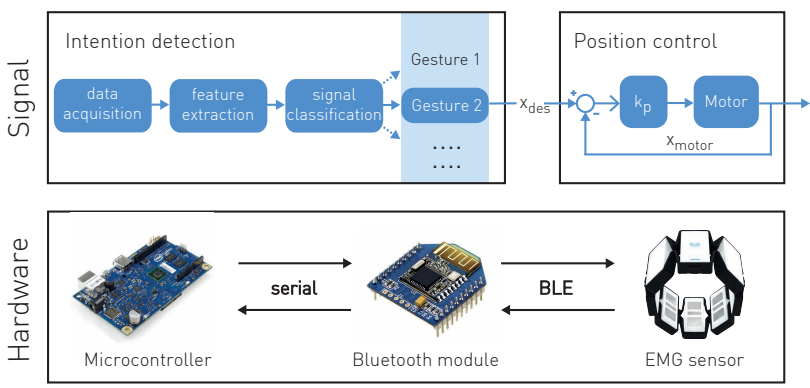

Fig. 4. Control scheme for the allocation of the EMG samples. On the hardware side, the signal pathway starts on the microcontroller with $\mathrm{C}$ commands that are translated into Bluetooth specific requests (UUID), sent over a serial connection to the Bluetooth module and wirelessly to the Myo armband, where the requests are processed and the responses are sent back in reversed order. The raw EMG samples are then processed (feature calculation) and classified, and depending on the assigned gesture, another action can be triggered, e.g. a new desired motor position $x_{d e s}$ of the robotic hand orthosis can be approached.

another XBee module, e.g. Zigbee, if a wireless connection to a computer is required. The Bluetooth module is based on Texas Instruments CC2541 System-on-Chip. The module acts as master to write status commands to the Myo armband and subscribe for notifications to get new EMG data. Instead of the custom firmware, the open source project myobridge [27] was flashed onto the HM-11 Bluetooth module. Myobridge contains an implementation of Thalmic's Myo armband Bluetooth protocol [28] to translate Bluetooth specific commands into Arduino compatible commands and vice versa (Fig. 4). The myobridge firmware was adjusted to fit the data structure alignment of the Intel processor on the Galileo Gen2.

\section{Signal processing and classifier}

As embedded systems provide a limited amount of computational power, the optimal trade-off between the computational cost of the classifier and its performance had to be found. We chose a multiclass support vector machines approach with segmentation lengths of $300 \mathrm{~ms}$, and a subset of the EMG time-domain features proposed by Englehart [24]: The mean absolute value $\left(F_{\text {mav }}=\frac{1}{S} \sum_{k=1}^{S}\left|x_{k}\right|\right)$, number of zero-crossings $\left(F_{z c}=\sum_{k=2}^{S}|1|\right.$ if $\left.\left(x_{k} * x_{k-1}\right)<0\right)$ and the waveform length $\left(F_{w l}=\sum_{k=2}^{S}\left|x_{k}-x_{k-1}\right|\right)$, where $\mathrm{x}$ is the EMG amplitude and $\mathrm{S}$ the length of the segmentation window. A radial basis kernel $\left(K\left(x_{i}, x_{j}\right)=\exp \left(-\gamma \| x_{i}-\right.\right.$ $\left.\left.x_{j} \|^{2}\right), \gamma>0\right)$ was used, where the kernel parameters $(\gamma, C)$ were optimized with a grid search. The multi-class problem was addressed with one-vs-one binary classifiers. For the online classification the sliding window was reduced to a length of $150 \mathrm{~ms}$ and shifted by $20 \mathrm{~ms}$ to reduce the delay. The features were normalized by the EMG value corresponding to maximal voluntary contraction (MVC) when closing the hand. The classification algorithm was implemented in Matlab for offline analysis and in Arduino IDE for the real-time embedded system. The Arduino IDE offers no classification library. Thus, we included the LIBSVM library [29], an

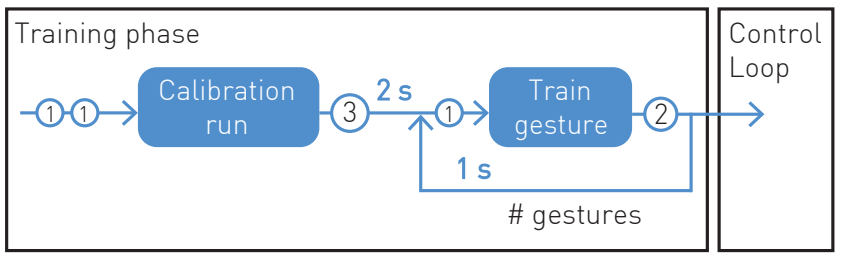

Fig. 5. Routine for the training of the classifier: The circles indicate short (1), medium (2) or long vibrations (3). During the calibration run, the maximal voluntary contraction signal is recorded. Then, for each gesture, a set of training samples is recorded for 2 seconds. After the training phase, the software enters the online classification loop. For three gestures, the entire training routine requires approximately 20 seconds.

integrated software for support vector classification with an efficient open source implementation in C. The classifier remained unchanged, however, we adapted the underlying functions of the framework to run it with the embedded system.

\section{E. Training interface}

Each time the Myo armband is donned a new classifier has to be trained wherefore a training interface with haptic feedback was implemented. Before the training routine starts, the Myo armband is placed on the forearm (see Fig. 1) and is set in non-sleep mode to prevent the armband from falling into a resting state. The streaming of the EMG is enabled and the built-in classifier is disabled. The initialization of the Myo armband includes a calibration run to measure the EMG values during MVC and the training of the classifier (Fig. 5). To guide the user through the routine (e.g. when the calibration ends and when a new training gesture should be performed), the vibration motor in the Myo armband is used.

\section{F. Validation procedure}

1) Offline classification accuracy for different gestures: To validate if the chosen classification approach meets the requirement of detecting five different hand gestures, its classification accuracy was offline analyzed. EMG data were collected with the Myo armband from one unimpaired subject with a streaming rate of $50 \mathrm{~Hz}$. The evaluation sets consisted of data from five static gestures (rest, close, open, precision pinch and key pinch, Fig 6), each held for one minute. In Matlab, a tenfold cross validation was performed for each evaluation set, i.e. 9/10th of the data acquired from each gesture were used to train a classification model while the remaining data was used to evaluate the classifier. The classification accuracy was computed as the number of correctly classified samples divided by the total number of samples.

2) Online classification accuracy for different arm configurations: To evaluate the applicability of the classifier in tasks of daily living, i.e. the recognition of different grasps in situations which differ from the training condition, the realtime classification accuracy was measured for three gestures, which can be executed with the hand orthosis presented 


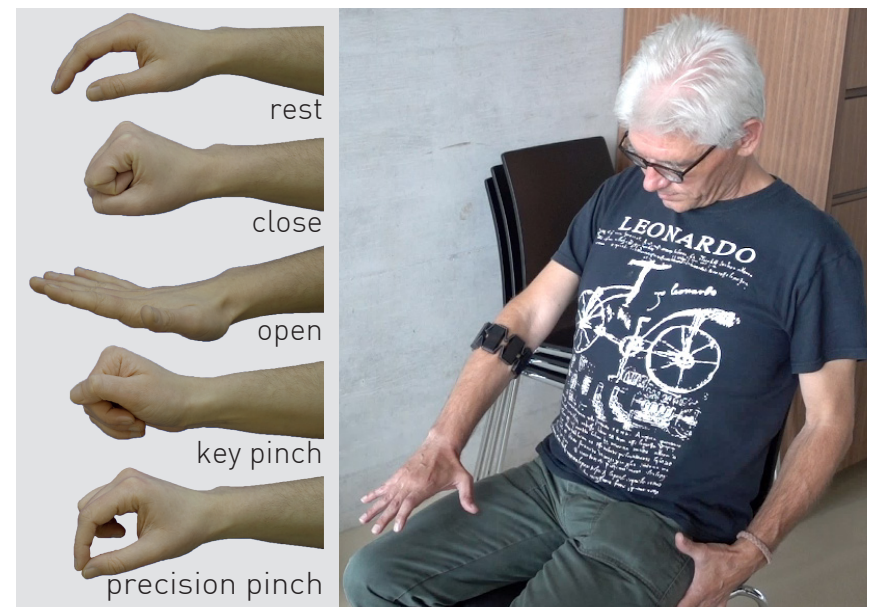

Fig. 6. On the left side, the hand gestures tested with the Myo armband are shown: rest, close, open, key pinch and precision pinch. On the right side, a stroke survivor wearing the Myo armband on the impaired side is performing an opening gesture.

TABLE I

UPPER EXTREMITY FUGL-MEYER SCORES OF THE THREE STROKE SURVIVORS

\begin{tabular}{|l|c|c|c|}
\hline Upper extremity Fugl-Meyer score & Patient 1 & Patient 2 & Patient 3 \\
\hline Proximal (maximum 36) & 12 & 26 & 28 \\
\hline Distal (maximum 30) & 2 & 16 & 23 \\
\hline Total (maximum 66) & 14 & 42 & 51 \\
\hline
\end{tabular}

in [20] (rest, close, open) in three arm configurations: (1) a relaxed position where the arm lays on the knee (training position), (2) stretching the arm in front of the body and (3) stretching the arm to the right of the body (Fig. 7). Evaluation data were collected from one unimpaired subject with a streaming rate of $50 \mathrm{~Hz} .150$ samples acquired in configuration 1 were used to train the classifier. To test the classification accuracy, the performance over three seconds per gesture in each arm configuration was analyzed, whereas the transient signal between two gestures was removed. This procedure was repeated 5 times, generating 5 different datasets.

3) Offline classification accuracy for stroke survivors: For a proof-of-concept, three male stroke survivors with mildly to severely impaired hand function on their right side (upper extremity Fugl-Meyer (FM) scores [30] of 14, 42 and 51 out of maximum 66 (Table I)) participated in a study, approved by the cantonal ethics commission (Approval No. Req-201600656). Following the FM upper limb assessment, EMG data were collected from the impaired arm with a streaming rate of $50 \mathrm{~Hz}$. The stroke survivors performed three gestures (rest, close, open, see Fig. 6), each gesture held for sixty seconds in arm configuration 1. The EMG data was offline processed, i.e. features were calculated, normalized and evaluated with tenfold cross validation.

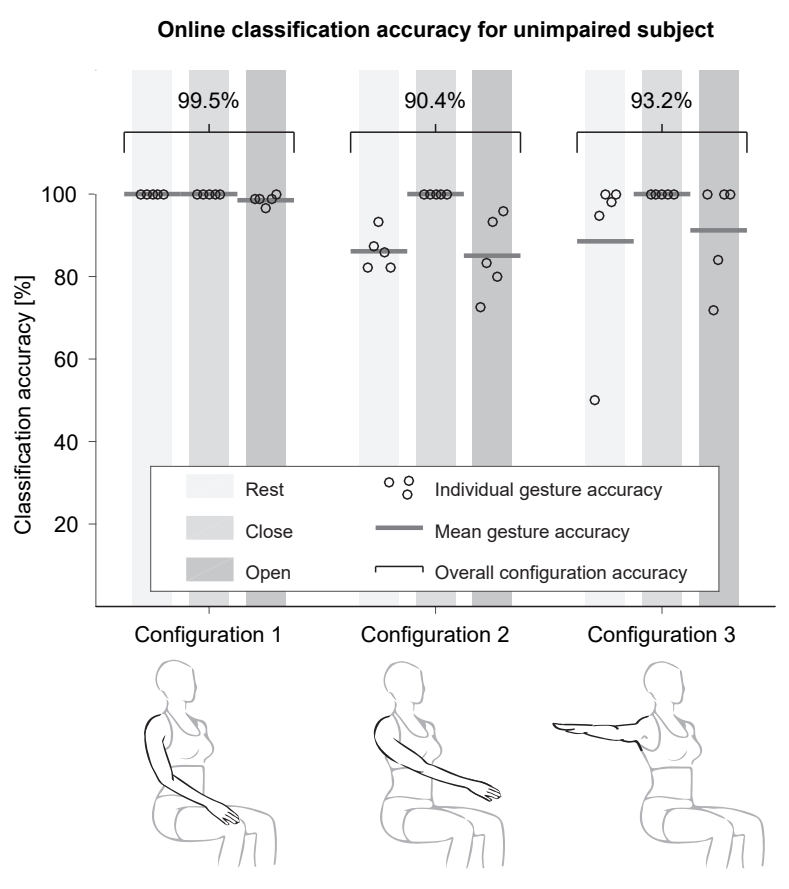

Fig. 7. Classification accuracies for the online evaluation with one unimpaired subject. Five datasets were recorded for three gestures in three different arm configurations. The grey shaded areas indicate the performed gesture (rest, open, close). The black circles denote accuracies for specific gestures in single datasets. The dark grey bars indicate the mean classification accuracy for specific gestures over all datasets. The black braces summarize the overall classification accuracies for each arm configuration (1: relaxed position, training position, 2: stretching the arm out in front of the body and 3: stretching the arm out to the right of the body).

\section{RESUlts}

1) Offline classification accuracy for different gestures: For the SVM approach, cross validated classification accuracies of over $98 \%$ were achieved for all 5 gestures.

2) Online classification accuracy for different arm configurations: The results of the online classifier analysis over different arm configurations are shown in figures 7 and 8. Mean classification accuracies of over $98 \%$ for each gesture (mean gesture accuracy) were achieved if the arm was held in configuration 1 (training position). When the arm was extended to other arm configurations $(2,3)$, the mean gesture accuracies lied between $85-100 \%$. The overall configuration accuracy (mean of the mean gesture accuracy for each arm configuration) varied between $90.4-99.5 \%$. The global classification accuracy was found to be $94.3 \%$. The classifiers detect the closing gesture with no false positives over all datasets, while the rest and opening gestures were mistaken as closing gesture in $2.3 \%$ to $3.3 \%$ of all cases, respectively.

3) Offline classification accuracy for stroke survivors:

The offline classification model for three gestures (rest, close and open) provided overall classification accuracies of $98.2 \%, 78.8 \%$ and $98.2 \%$ for each patient respectively (Fig. 9).

4) System usability: The stroke survivors wore the Myo armband for 10 to 20 minutes reporting comfortable use, also during the vibrating feedback of the armband. With 
Online classification confusion matrix for unimpaired subject

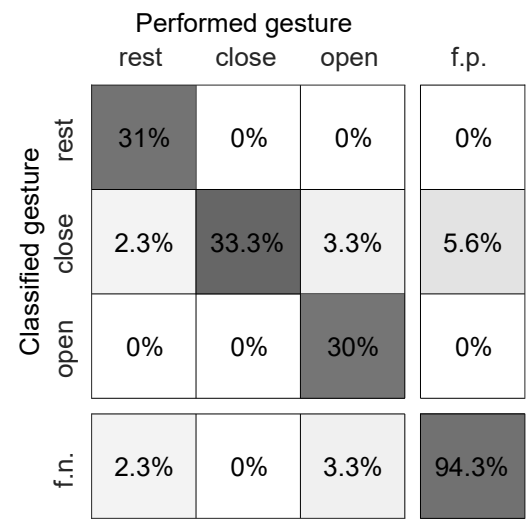

Fig. 8. Confusion matrix for the online classification. For rest, close and open, the number in the boxes indicate the percentage of all signal segmentations classified as the corresponding gesture. In the right column (f.p.) the false positives (i.e. samples classified as the corresponding gesture, when actually another gesture was performed) are indicated. The row f.n indicates the false negatives (i.e. the relative amount of incorrectly classified samples of the corresponding gesture).

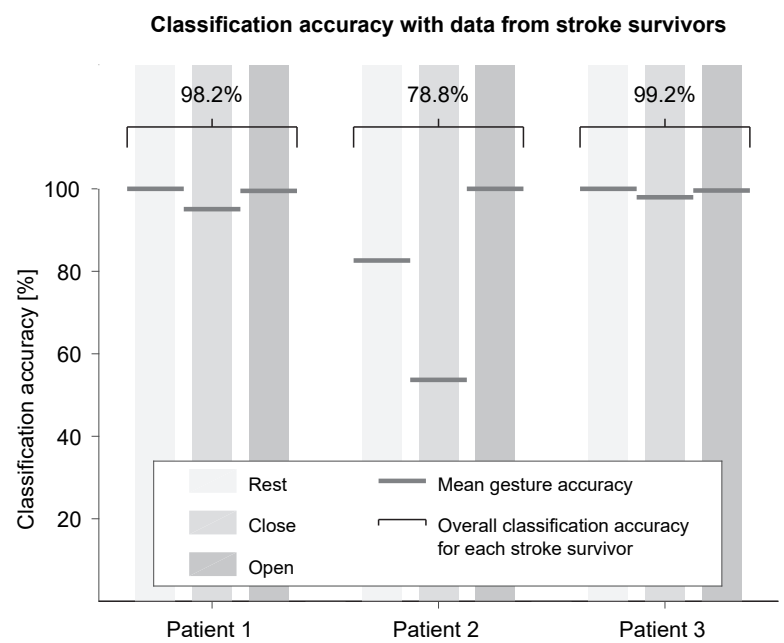

Fig. 9. Classification accuracies for the offline evaluation with three stroke survivors (upper extremity FM scores of 14, 42 and 51 out of max. 66). Data were recorded for three gestures, each gesture held for sixty seconds in a relaxed arm configuration with the arm on the knee. The grey shaded areas indicate the performed gesture (rest, open, close). The dark grey bars indicate the mean classification accuracy for specific gestures. The black braces summarize the overall classification accuracies for each stroke survivor.

a segment length of 30 samples, a delay of $600 \mathrm{~ms}$ for a streaming frequency of $50 \mathrm{~Hz}$ is achieved. The processing time of the classification itself is in the range of a few milliseconds and therefore negligible. The training routine starts automatically, lasting for 20 seconds. The system allows continuous classification for up to $4 \mathrm{~h}$ on a single battery charge. It was successfully integrated into a hand exoskeleton [20] and used as embedded myoelectric control for the wearable robotic hand orthosis.

\section{Discussion}

We presented an embedded myoelectric intention detection system using the Myo armband for EMG data acquisition.
The classifier is customizable with a training routine guiding through the training phase. The algorithm was evaluated with respect to different arm configurations and tested with data from stroke survivors.

\section{A. System characteristics and advantages}

The proposed system is fully wearable and stand-alone, including the hard and software for real-time EMG intention detection and it is able to control a robotic hand orthosis. To the authors' knowledge, only one similar stand-alone intention detection system with the Myo armband has been developed: Dicker et al. [31] described the intended use with hand prosthesis. However, neither online classification accuracy nor robot control were reported.

Compared to conventional EMG data acquisition systems (as used in [15]-[17]) the Myo armband is highly convenient to use. The dry electrodes do not require exact placement and can be placed all at once, such that stroke survivors themselves can don the Myo armband. The electronics board is small and can be well integrated into a robotic device.

Once the Myo armband is donned, the user is guided through a training routine, where the classifier is trained with desired gestures. Such training is necessary for every state of the art EMG system [15]-[18], as EMG is not an absolute measure and changes for different users and different sessions (electrode placement, fatigue of the user, etc.) [21]. If the myoelectric intention detection system is used to control a robotic device, the complete setup from donning the Myo armband to the start of the real-time control of the device takes approximately 2 minutes including the training of the classifier (in comparison placing the Myo armband and training the built-in classifier requires approximately three minutes, whereas in state of the art EMG systems with individual electrodes [16], already the electrode placement requires several minutes). The user interface based on vibrating feedback, which guides the user through the training routine, and the subsequent embedded online classification fully decouple the system from stationary computers and displays. Furthermore, the rechargeable batteries allow for a use of several hours, making the proposed system suitable for independent use in the home environment.

\section{B. Classification algorithm and accuracy}

Out of various classifiers and features presented in [15], [24], [32], the best performing combination for this work was found iteratively. With the setup presented in section II we reached offline classification accuracies for 5 gestures of over $98 \%$, a comparable result to related work using state of the art EMG acquisition systems from Choi et al. of $97 \%$ for 5 gestures [33], Khokhar et al. reporting $96 \pm 1.98 \%$ for 13 gestures [34] or Dicker et al. with accuracies of $99 \pm 1 \%$ for 6 gestures [31]. Compared to related work presenting either integrated systems or use of the Myo armband, the presented system performs comparable: Benatti et al. [16] used conventional EMG electrodes and a SVM classifier in a fully embedded system (without the option for embedded classifier training) and reached slightly lower accuracies of 
$92 \%$ but for an increased number of 7 different hand gestures. Abreu et al. [32] used the LIBSVM library to train a SVM classifier with EMG from the Myo armband and reached offline classification accuracies of $4-95 \%$ for 20 different hand gestures. In order to increase the acceptance as a therapeutic device, we prioritize to recognize the performed gesture with reliable accuracy over implementing a high number of grasps.

For the three gestures evaluated in the online classification the proposed system reaches classification accuracies of $85-100 \%$, comparable to results found in literature (Fougner et al. $95 \%$ for 8 gestures with training data from different arm configurations [35], Benatti et al. 90\% accuracy for 7 gestures [16]). We can see that the online classification accuracy varies for different gestures and different datasets. The former can be explained with the much higher EMG amplitude of the closing gesture compared to other gestures. This biases the classifier towards the closing gesture: every time the performed resting and opening gestures were misclassified they were classified as closing gesture, whereas no actually performed closing gesture was misclassified. The variation in classification accuracy for different datasets can be explained by differences in the quality of the training data, introduced when the user does not follow the training routine or performs gestures differently during training and classification.

For certain arm configurations the classification accuracy for single gestures in single datasets can decrease down to $50 \%$. Much like the varying classification accuracy for different gestures and datasets, this is due to the low variance in the training set. The classifier can be made more robust for signal changes by extending the classifier training routine in order to acquire more diverse training data or by reducing the number of extracted features.

\section{Performance in stroke survivors}

Related work presenting hand gesture recognition in stroke survivors based on state of the art EMG systems report classification accuracies of $92.42 \pm 5.51 \%$ for 20 gestures [18] or $71.3 \%$ for six gestures in a study with mildly impaired subjects [21]. In this work, we evaluated data from mildly to severely impaired subjects for three gestures. The classification accuracy between $78 \%$ and $98.5 \%$ is comparable to the results found in related work.

For subject 3 high classification accuracies were reached for all three gestures. This result was expected, as subject 3 was only mildly impaired. The low classification accuracy for subject 2 (moderately impaired) is due to imprecisely followed oral instructions during the data acquisition, which led to differently performed gestures and high variance in the EMG data. This highlights again that the classification accuracy strongly depends on the quality of the training data, especially for a short training routine. Remarkable is the classification accuracy reached for subject 1: despite the severe impairment of the hand (distal upper extremity FM score of only 2 out of max. 30) and almost no residual motion when attempting to perform the three gestures, the EMG signal of subject 1 could be correctly classified in over $98 \%$ of all cases. While limited to a single subject, this highlights the potential of the presented system for EMG based robot control and therefore robot assisted home rehabilitation for stroke survivors.

\section{Limitations}

The presented results were acquired in a lab environment and the use of the system in activities of daily living has to be validated. Furthermore, in stroke survivors, the system has neither been tested for online classification nor for different arm configurations, mostly due to low residual proximal limb mobility of the participating stroke survivors. Thus, the presented results for offline classification in stroke survivors may not be fully representative.

Besides the pending validation of the results, some technical limitations have to be taken into account. In the current configuration, the total delay of the system from intended motion of the user until execution of the motion of the robotic hand orthosis is $600 \mathrm{~ms}$, which can be perceived by the user. In comparison with other EMG systems with delays of only $125 \mathrm{~ms}$ for acquisition and processing [36], this is a clear limitation of the proposed system. However, with the maximal streaming rate of $200 \mathrm{~Hz}$ of the Myo armband (limited by the bandwidth of the BLE protocol) and the need for 30 samples to correctly classify the performed gestures, the theoretical minimal delay is $150 \mathrm{~ms}$, which would lay below the perceivable limit of 250-300ms [24]. Additionally, the transient EMG signal generated when changing between two gestures, might be included to further shorten the classification delay.

A further limitation is the misclassification of single samples: if one recorded sample is assigned to the wrong class, the robot reacts instantly, which leads to a twitching motion of the robotic device. This would largely limit applications in activities of daily living. In order to filter misclassified single samples, majority vote can be integrated (i.e. the motion of the robot is only triggered, when a new gesture is recognized continuously over several samples) at the expense of an increased delay.

For a future use, the robustness of the signal transfer will be improved, and a more elaborated error handling routine will be included to increase the acceptance of the system. To reduce arm configuration dependency of the gesture classification, data from different arm configurations as well as data from inertial measurement units (IMU) can be integrated in the training. Further evaluation of the realtime controlled system with patients should be conducted. A representative study is planned to specify the subset of stroke survivors who could use the EMG triggered robotic hand orthosis and benefit from its assistance.

\section{CONCLUSiON}

In this paper we presented a stand-alone EMG-based intention detection system including a fast training routine using the Myo armband. We showed that this system can 
be used for gesture recognition of five custom hand gestures (rest, close, open, precision pinch and key pinch) in healthy subjects and three gestures (rest, close, open) in stroke survivors. The classifier reached accuracies of $78.8 \%$ to $99.2 \%$ for mildly to severely impaired subjects, and as a proof-of-concept, the classification output was used to control a robotic hand orthosis, indicating the potential of myoelectric control for wearable robotic hand orthoses in home rehabilitation.

\section{ACKNOWLEDGEMENTS}

This work is supported by the Swiss National Science Foundation through the National Centre of Competence in Research on Robotics. The authors would like to thank Professor Nicole Wenderoth, Charles Lambelet and Mingxing Lyu for the inspiring discussions. The authors would also like to thank Mike D. Rinderknecht for providing his Matlab Plot Toolbox and the patients who volunteered for the study.

\section{REFERENCES}

[1] J. J. Guilbert, "The world health report 2002 - reducing risks, promoting healthy life." Education for health (Abingdon, England), vol. 16, no. 2, p. 230, 2003.

[2] H. Fischer, K. Stubblefield, T. Kline, X. Luo, R. Kenyon, and D. Kamper, "Hand Rehabilitation Following Stroke: A Pilot Study of Assisted Finger Extension Training in a Virtual Environment," Topics in Stroke Rehabilitation, vol. 14, no. 1, pp. 1-12, 2007.

[3] K. Ottenbacher and S. Jannell, "The results of clinical trials in stroke rehabilitation research," Archives of Neurology, vol. 50, no. 1, pp. 3744, jan 1993.

[4] E. Taub and A. Berman, Movement and learning in the absence of sensory feedback. Dorsey Press Homewood, Illinois, 1968.

[5] G. Kwakkel, "Intensity of practice after stroke: More is better," Schweizer Archiv fur Neurologie und Psychiatrie, vol. 160, no. 7, pp. 295-298, 2006.

[6] J. Ramirez, M. Alfaro, and I. Chairez, "Electromyographic Driven Assisted Therapy for Hand Rehabilitation by Robotic Orthosis and Artificial Neural Networks," in IFMBE Proceedings, vol. 49, 2015, pp. $75-78$.

[7] J. Iqbal, O. Ahmad, and A. Malik, "HEXOSYS II - Towards realization of light mass robotics for the hand," Proceedings of the 14th IEEE International Multitopic Conference 2011, INMIC 2011, pp. 115-119, 2011.

[8] S. Ates, I. Mora-Moreno, M. Wessels, and A. H. A. Stienen, "Combined active wrist and hand orthosis for home use: Lessons learned," in IEEE International Conference on Rehabilitation Robotics, 2015, pp. 398-403.

[9] V. Dietz, T. Nef, and W. Z. Rymer, Neurorehabilitation Technology. Springer Science \& Business Media, 2011.

[10] S. Löwel and W. Singer, "Selection of intrinsic horizontal connections in the visual cortex by correlated neuronal activity," Science, vol. 255, no. 5041, pp. 209-212, 1992.

[11] L. Randazzo, I. Iturrate, R. Chavarriaga, R. Leeb, and J. D. R. Millan, "Detecting intention to grasp during reaching movements from EEG," in Proceedings of the Annual International Conference of the IEEE Engineering in Medicine and Biology Society, EMBS, 2015, pp. 11151118.

[12] S. R. Soekadar, M. Witkowski, N. Vitiello, and N. Birbaumer, "An EEG/EOG-based hybrid brain-neural computer interaction (BNCI) system to control an exoskeleton for the paralyzed hand," Biomedizinische Technik, vol. 60, no. 3, pp. 199-205, 2015.

[13] T. Nagaoka, K. Sakatani, T. Awano, N. Yokose, T. Hoshino, Y. Murata, Y. Katayama, A. Ishikawa, and H. Eda, "Development of a New Rehabilitation System Based on a Brain-Computer Interface Using Near-Infrared Spectroscopy Takafumi," Advances in Experimental Medicine and Biology, vol. 662, pp. 7-25, 2010.

[14] T. Feix, J. Romero, H. B. Schmiedmayer, A. M. Dollar, and D. Kragic, "The GRASP Taxonomy of Human Grasp Types," IEEE Transactions on Human-Machine Systems, vol. 46, no. 1, pp. 66-77, 2015.
[15] M. Atzori, H. Muller, and M. Baechler, "Recognition of hand movements in a trans-radial amputated subject by sEMG," IEEE International Conference on Rehabilitation Robotics, 2013.

[16] S. Benatti, F. Casamassima, B. Milosevic, E. Farella, P. Schönle, S. Fateh, T. Burger, Q. Huang, and L. Benini, "A Versatile Embedded Platform for EMG Acquisition and Gesture Recognition," IEEE Transactions on Biomedical Circuits and Systems, vol. 9, no. 5, pp. 620-630, 2015.

[17] R. Gopura and K. Kiguchi, "A human forearm and wrist motion assist exoskeleton robot with EMG-based fuzzy-neuro control," Proceedings of the 2nd Biennial IEEE/RAS-EMBS International Conference on Biomedical Robotics and Biomechatronics, BioRob 2008, pp. 550$555,2008$.

[18] X. Zhang and P. Zhou, "Classification of upper limb motions in stroke using high density surface EMG," in 2011 Annual International Conference of the IEEE Engineering in Medicine and Biology Society, 2011, pp. 3367-3370.

[19] S. Masson, F. S. Fortuna, F. S. Moura, and D. C. Soriano, "Integrating Myo Armband for the Control of Myoelectric Upper Limb Prosthesis," in XXV Congresso Brasileiro de Engenharia Biomédica - CBEB 2016, 2016, pp. 1-4.

[20] C. J. Nycz, T. Butzer, O. Lambercy, J. Arata, G. S. Fischer, and R. Gassert, "Design and Characterization of a Lightweight and Fully Portable Remote Actuation System for Use with a Hand Exoskeleton," IEEE Robotics and Automation Letters, vol. 1, no. 2, pp. 976-983, 2016.

[21] S. W. Lee, K. M. Wilson, B. A. Lock, and D. G. Kamper, "Subjectspecific myoelectric pattern classification of functional hand movements for stroke survivors," IEEE Transactions on Neural Systems and Rehabilitation Engineering, vol. 19, no. 5, pp. 558-566, 2011.

[22] M. Santello, M. Flanders, and J. F. Soechting, "Postural hand synergies for tool use," The Journal of Neuroscience, vol. 18, no. 23, pp. 10105 $10115,1998$.

[23] A. Fougner, E. Scheme, A. D. C. Chan, K. Englehart, and Ø. Stavdahl, "Resolving the Limb Position Effect in Myoelectric Pattern Recognition," IEEE Transactions on Neural Systems and Rehabilitation Engineering (2011), vol. 19, no. 6, pp. 644-651, 2011.

[24] K. Englehart and B. Hudgins, "A robust, real-time control scheme for multifunction myoelectric control," IEEE Transactions on Bio-Medical Engineering, vol. 50, no. 7, pp. 848-54, 2003.

[25] "Myo Gesture Control Armband - Wearable Technology by Thalmic Labs." [Online]. Available: https://www.myo.com/

[26] "BLE Bee - Wiki." [Online]. Available: http://wiki.seeedstudio.com/ wiki/BLE $\left\{{ }_{-}\right\}$Bee

[27] V. Roland, "Arduino library and firmware for HM-11." [Online] Available: https://github.com/vroland/MyoBridge

[28] "Thalmic Labs Bluetooth protocol for Myo armband." [Online] Available: https://github.com/thalmiclabs/myo-bluetooth

[29] C.-C. Chang and C.-J. Lin, "Libsvm," ACM Transactions on Intelligent Systems and Technology, vol. 2, no. 3, pp. 1-27, 2011. [Online]. Available: http://dl.acm.org/citation.cfm?doid=1961189.1961199

[30] A. R. Fugl-Meyer, L. Jääskö, I. Leyman, S. Olsson, and S. Steglind, "The post-stroke hemiplegic patient. 1. a method for evaluation of physical performance." Scandinavian journal of rehabilitation medicine, vol. 7, no. 1, pp. 13-31, 1975.

[31] O. Dicker and A. Peleg, Real Time Control of Hand Prosthesis Using $E M G, 2016$.

[32] J. Abreu, J. Teixeira, and L. Figueiredo, "Evaluating Sign Language Recognition Using the Myo Armband," in Virtual and Augmented Reality (SVR), 2016 XVIII Symposium on, 2016.

[33] C. Choi and J. Kim, "A real-time EMG-based assistive computer interface for the upper limb disabled," in 2007 IEEE 10th International Conference on Rehabilitation Robotics, ICORR'07, 2007, pp. 459462.

[34] Z. O. Khokhar, Z. G. Xiao, C. Menon, and Others, "Surface EMG pattern recognition for real-time control of a wrist exoskeleton," Biomedical engineering online, vol. 9, no. 1, p. 41, 2010.

[35] A. Fougner, E. Scheme, S. Member, A. D. C. Chan, and S. Member, "A Multi-Modal Approach for Hand Motion Classification Using Surface EMG and," in 33rd Annual International Conference of the IEEE EMBS Boston, Massachusetts USA, 2011, pp. 4247-4250.

[36] J. U. Chu, I. Moon, and M. S. Mun, "A real-time EMG pattern recognition system based on linear-nonlinear feature projection for a multifunction myoelectric hand," IEEE Transactions on Biomedical Engineering, vol. 53, no. 11, pp. 2232-2239, 2006. 